\title{
Die Sicherheit des Lebendnierenspenders - Das Deutsche Lebendspende Register SOLKID-GNR - Entstehung und Struktur eines nationalen Registers in der Versorgungsforschung
}

\section{Safety of the Living Kidney Donor - The German National Register - Development and Structure of a National Register in the Health Service Research}

\section{Autoren}

Barbara Suwelack', Martin Dugas², Martina Koch ${ }^{3}$, Claudia Sommerer ${ }^{4}$, Marc Urban ${ }^{5}$, Joachim Gerß ${ }^{6}$, Jeannine Wegner ${ }^{1}$, Markus Burgmer7,8

\section{Institute}

1 Medizinische Klinik D - Transplantationsnephrologie, Universitätsklinikum Münster, Münster, Deutschland

2 Institut für Medizinische Informatik, Westfälische Wilhelms-Universität Münster, Münster, Deutschland

3 Klinik für Allgemein-, Universitätsmedizin der Johannes Gutenberg-Universität Mainz, Viszeral- und Transplantationschirurgie, Mainz, Deutschland

4 Nephrologie am Zentrum für Innere Medizin, UniversitätsKlinikum Heidelberg, Heidelberg, Deutschland

5 Zentrum für Klinische Studien, Westfälische WilhelmsUniversität Münster, Münster, Deutschland

6 Institut für Biometrie und Klinische Forschung, Westfälische Wilhelms-Universität Münster, Münster, Deutschland

7 Abteilung für Psychosomatische Medizin und Psychotherapie, LWL-Klinik Münster, Münster, Deutschland

8 Klinik für Psychosomatik und Psychotherapie, Universitätsklinikum Münster, Münster, Deutschland

\section{Schlüsselwörter}

Lebendspender, Lebendnierenspende, Register, SOLKIDGNR, Transplantationsnephrologie

\section{Key words}

Living donor, Living kidney donation, Transplantation nephrology

\section{Bibliografie}

Gesundheitswesen 2021; 83(Suppl. 1): S33-S38

DOI 10.1055/a-1547-7114

ISSN 0949-7013

(c) 2021. Thieme. All rights reserved.

Georg Thieme Verlag, Rüdigerstraße 14,

70469 Stuttgart, Germany
Korrespondenzadresse

Prof. Dr. med. Barbara Suwelack

Medizinische Klinik D - Transplantationsnephrologie

Universitätsklinikum Münster

Münster

Deutschland

barbara.suwelack@ukmuenster.de

\section{ZUSAMMENFASSUNG}

Im Deutschen Lebendspende Register SOLKID-GNR (Safety of the Living Kidney Donor - The German National Register) werden medizinische und psychosoziale Daten zum Outcome von Lebendnierenspender erhoben. Die prospektive Datenerhebung ermöglicht erstmals in Deutschland eine wissenschaftlich fundierte Langzeitanalyse, wie sich eine Lebendnierenspende auf die psychische und physische Gesundheit von Lebendnierenspendern auswirkt. Dies trägt direkt zu einer Verbesserung der Information und Versorgung der Lebendnierenspender bei.

\begin{abstract}
The German living donor register Safety of the Living Kidney Donor - The German National Register (SOLKID-GNR) collects data of the medical and psychosocial outcome of living kidney donors. For the first time in Germany, a prospective data collection allows a scientifically based long-term analysis of how a living kidney donation influences the psychological and physical health of living kidney donors. This will contribute directly to improve the information and care of living kidney donors.
\end{abstract}




\section{Einleitung}

Die Nierentransplantation (NTx) stellt die optimale Therapie des terminalen chronischen Nierenversagens dar. Aufgrund des besonders in Deutschland bestehenden Organmangels und der damit verbundenen zunehmenden Wartezeit auf eine Spenderniere eines Verstorbenen ist die freiwillige Lebendnierenspende (LS) nach dem Transplantationsgesetz eine alternative Therapieoption. Der Anteil der LS beträgt in Deutschland 30\% der NTx. Aufgrund der langen Wartezeiten besteht ein nicht unerheblicher psychosozialer Druck auf die Lebendnierenspender (LNS) eine Niere zu spenden und so dem Empfänger die langen Wartezeiten und die Dialyse zu ersparen. Auch der Anteil älterer LNS mit Begleiterkrankungen - sog. marginale Spender - wächst stetig. Ferner ist aber auch eine Verunsicherung hinsichtlich negativer Konsequenzen für die Lebensqualität zu beobachten. Weltweite klinische Erfahrungen gingen bisher davon aus, dass die LS mit einem für den LNS akzeptablen minimalen Risiko einhergeht. In der Literatur finden sich aber auch meist retrospektive monozentrisch erhobene deutsche Studien, die z.T. Hinweise auf eine Verschlechterung der Lebensqualität (Quality of Life, QoL) nach LS gefunden haben [1,2]. Leider gibt es bis heute keine belastbaren Ergebnisse zum Einfluss einer LS auf das kombinierte physische und psychosoziale Ergebnis der LNS. Insbesondere das heute zu beobachtende geänderte LNS-Profil könnte das Risiko erhöhen. Da kein systematisches Transplantations-, geschweige Lebendspende-Register zum psychosozialen, sozioökonomischen und medizinischen Outcome der LNS in Deutschland existiert, basieren die aktuellen Empfehlungen auf retrospektiven Daten anderer Länder, anderer Gesundheitssysteme, Gesetzgebungen und anderer Bevölkerungscharakteristika [3-8]. US amerikanische und norwegische Register-Daten zeigten, dass LNS weniger günstige Langzeitverläufe als erwartet aufwiesen und eine z.T. eingeschränkte Lebenserwartung nach Lebendnierenspende haben [9-12].

Das Gesamtziel des Registers ist daher die Verbesserung der Einschätzung der medizinischen und psychosozialen Spenderrisiken im Langzeitverlauf im deutschen Gesundheitssystem durch die systematische und prospektive Datenerfassung vor und nach LS. Anhand einer detaillierten prospektiven Analyse der Register-Daten soll bereits vor der Spende diejenigen LNS identifiziert werden, die ein erhöhtes bzw. zu hohes Risiko für einen negativen psychosozialen und medizinischen Outcome haben. Bei den betroffenen potentiellen LNS kann somit die Aufklärung und der Einwilligungsprozess individuell angepasst werden und maßgeschneiderte Maßnahmen zur Risikoreduktion, implementiert werden.

Um wissenschaftlich fundierte Aussagen über den Verlauf und die mit der LS verbundenen möglichen Langzeitfolgen für LNS in Deutschland tätigen zu können, bedarf es eines systematischen, umfassenden und prospektiven Deutschen Lebendspende Registers, dessen Struktur wir hier vorstellen möchten.

\section{Registerstruktur}

Das Deutsche Lebendspende Register SOLKID-GNR (Safety of the Living Kidney Donor - The German National Register) wurde als Nachfolgeprojekt der in Münster initiierten SoLKiD Studie [13] als interdisziplinäres Register von drei Transplantationszentren (Münster, Heidelberg, Hamburg, jetzt Mainz) im Rahmen des BMBF (Bun- desministerium für Bildung und Forschung) Calls „Modellhafte Register - Konzeptentwicklungsphase“ (Förderkennzeichen: 01GY1725) beantragt. Das BMBF fördert seit dem 1. März 2019 eine 5-jährige Realisierungsphase des Registers (Förderkennzeichen: 01GY1906), deren Ziel es ist, eine über die Realisierungsphase hinaus, funktionierende Registerstruktur aufzubauen.

Das SOLKID-GNR beinhaltet verschiedene organisatorische Einheiten, die im Organigramm ( $>$ Abb. 1) zusammenfassend dargestellt sind.

Das Scientific Steering Committee (SSC), die Co-Principal Investigators und die Registerzentrale sind für die Implementierung, den Betrieb, der organisatorischen Durchführung des Registers und der wissenschaftlichen Analyse und Veröffentlichung der Registerdaten verantwortlich.

Die Registerzentrale setzt sich aus der Registerkoordination, dem Datenmonitoring, der Medizininformatik, der Biometrie und dem Datenmanagement zusammen, die an der Medizinischen Fakultät der Westfälischen Wilhelms-Universität (WWU) Münster lokalisiert sind. Die Registerzentrale ist die wesentliche Schnittstelle der Kommunikation zwischen allen Bereichen und Mitgliedern des Registerkonsortiums ( $\triangleright$ Abb. 1).

Um einen hohen wissenschaftlichen Standard im Register zu etablieren, wurden weitere nationale und internationale Experten aus der Psychosozialen Medizin, Chirurgie/Urologie und Nephrologie in das Registerkonsortium aufgenommen und bilden das Externe Scientific Advisory Board (eSAB), sowie das Interne Scientific Advisory Board (iSAB). Das eSAB steht dem SSC beratend zur Verfügung und dient der Sicherung der Qualität des Registers, der Datenauswertung sowie der politischen Akzeptanz und Etablierung. Das iSAB bildet eine wissenschaftliche Schnittstelle zwischen den Transplantationszentren (TZ) und dem SSC und besteht aus drei Vertretern der teilnehmenden TZ, die von den teilnehmenden TZ gewählt werden.

Durch die Einbindung der Patientenorganisationen Bundesverband Deutscher Organtransplantierter (BDO e.V.), Bundesverband Niere (BN e.V.) und der Selbsthilfe Lebendorganspende Deutschlands (SLOD e.V.) wird das Register bei Betroffenen bekannt und somit die Teilnahmebereitschaft der LNS am Register erhöht.

\section{Teilnehmende Transplantationszentren}

Am SOLKID-GNR nehmen 38 von 38 in Deutschland aktiven TZ, die LS durchführen, teil.

Die TZ sind verantwortlich für den prospektiven Einschluss möglichst aller LNS, die Aufklärung und das Einholen der Einwilligungserklärung der Spender, sorgen für die Aushändigung der elektronischen Fragebögen (Tablet-basiert), pflegen die klinischen Daten in die elektronischen Case Report Forms (eCRF, Arztfragebögen) ein und arbeiten mit der Registerzentrale zusammen.

\section{Register-Teilnehmer}

Ziel ist es, möglichst allen LNS, die in Deutschland eine Niere spenden werden, die Teilnahme am Register zu ermöglichen. Da aktuell nur LNS am Register teilnehmen können, die über ausreichende deutsch-Kenntnisse verfügen, weicht die Quellpopulation (alle LNS) gegenüber der tatsächlich ins Register aufgenommenen Anzahl an 


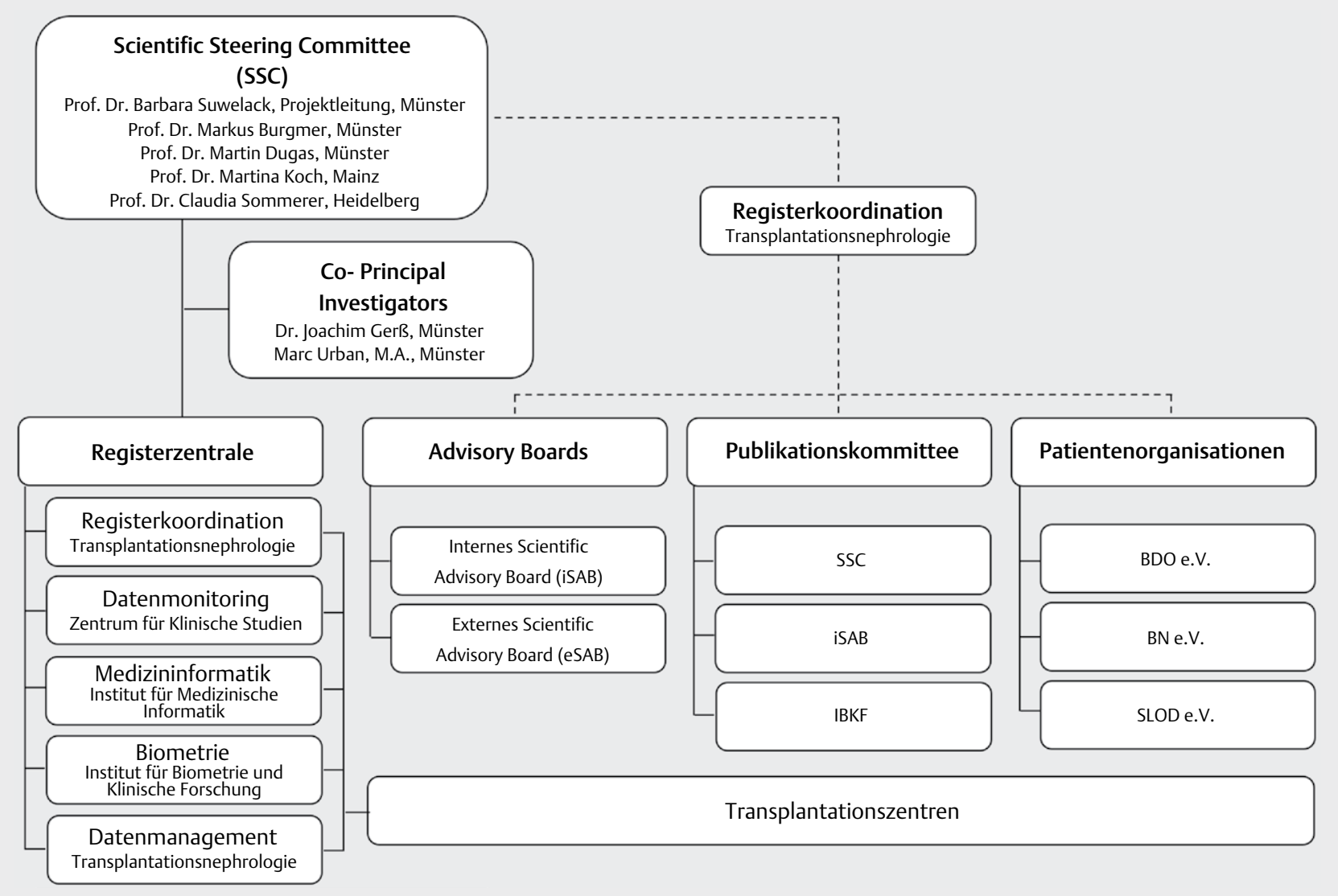

Abb. 1 Organigramm des SOLKID-GNR Konsortiums. Struktureller Aufbau und Organisationseinheiten des SOLKID-GNR. SSC=Steering Scientific Committee; iSAB = Internes Scientific Advisory Board; eSAB = Externes Scientific Advisory Board; IBKF=Institut für Biometrie und Klinische Forschung Münster; BDO e.V. = Bundesverband der Organtransplantierten; BN e.V. = Bundesverband Niere; SLOD e.V. = Selbsthilfeorganisation für Lebendorganspender.

LNS ab. Auch wird ein eher geringer Anteil der Teilnahme am Register nicht zustimmen. Somit ist derzeit von einer Einschlussquote von $80 \%$ auszugehen.

\section{Datenerhebung}

Das wesentliche Verfahren zur Datenerhebung wird auf elektronischem Wege sein. Hierzu wurden eCRFs für die klinischen Daten zusammengestellt sowie Spender-Fragebögen für die Erfassung der psychosozialen Spenderrisiken und psychischen und physischen Gesundheit entwickelt und in eine SOLKID-GNR-App eingebunden. Die elektronische Datenerhebung wird mit auf Tablets hinterlegter SOLKID-GNR-App und elektronischen Datenerfassungssystem („REDCap ${ }^{\circledR “, ~[14]) ~ f u ̈ r ~ d i e ~ B r o w s e r-b a s i e r t e ~ D a t e n e i n g a b e ~ d e r ~ k l i-~}$ nischen Daten ermöglicht.

Nach Initiierung der TZ werden die Daten zu den Messzeitpunkten max. 4 Wochen vor der Lebendnierenspende (T0-Prä), 8-14 Wochen nach der Lebendnierenspende (T0-Post) und dann im jährlichen Rhythmus nach Spende erhoben (T1, T2, T3...; siehe > Abb. 2).

Sobald ein neuer LNS eingeschlossen wurde, wird aus seinen identifizierenden Daten (IDAT: Vorname, Nachname und Geburtsdatum) über eine sogenannte „Mainzelliste“ [15] automatisiert ein spenderspezifisches Pseudonym generiert. Die Zuordnung vom Pseudonym zu den IDAT wird lokal an den zuständigen TZ mittels Pseudonymisierungsliste nachgehalten. Außerhalb der lokalen TZ kann kein Rückschluss vom Pseudonym auf den LNS gezogen werden.

Der Pseudonymisierungsservice stellt sicher, dass der gleiche LNS dem gleichen Pseudonym zugeordnet wird, auch wenn dieser das TZ wechseln sollte. Alle registerbezogenen medizinischen Daten (MDAT) werden nun anhand des Pseudonyms web-basiert über die eCRFs erfasst. Dies ist auch bei der Tablet-basierten Dateneingabe über die SOLKID-GNR-App der Fall. Das REDCap ${ }^{\circledR}$ System verfügt über die Funktionalität eines Audit Trails, um Änderungen an Dateneinträgen zu protokollieren.

Sollte ein Registerteilnehmer seine Einwilligung zur Teilnahme widerrufen, oder anderweitig aus dem Register ausscheiden (z. B. Tod, Nicht-Erscheinen zu den Nachsorgeterminen) kann dies elektronisch mittels des Registerabbruch-Formulars, das zusätzlich zu dem Spender- und Arzt-Fragebögen in der SOLKID-GNR-App hinterlegt wurde, an die Registerzentrale gemeldet werden, die daraufhin den Datensatz anonymisiert, oder auf Verlangen des LNS unwiderruflich löscht. 


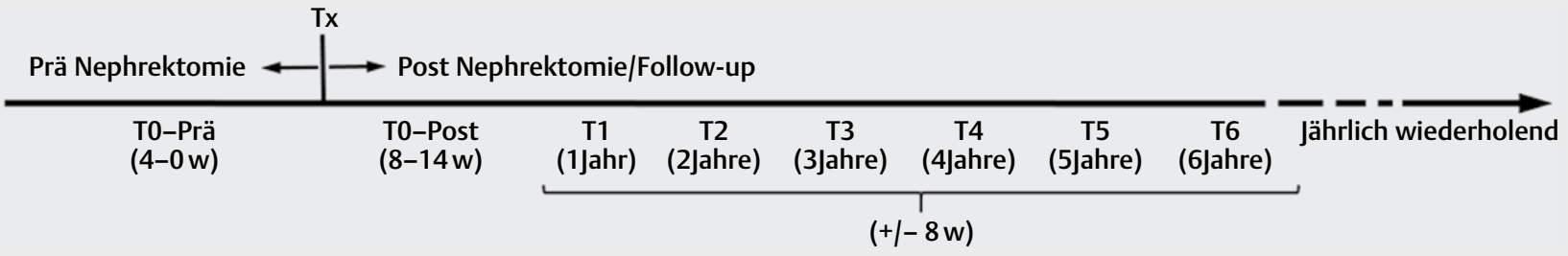

Abb. 2 Messzeitpunkte der Datenerhebung. Tx=Transplantation, w= Wochen.

Zur Verbesserung des Schutzes und zur Sicherstellung der Qualität der Registerdaten wurde entsprechend internationaler Standards und den Anforderungen der guten klinischen Praxis, eine risiko-basierte Strategie zur Qualitätskontrolle entwickelt und implementiert. Das SOLKID-GNR interne Monitoring prüft im Rahmen der Qualitätskontrolle die Abläufe um die informierte Einwilligung und die kritischen Registerparameter im Zuge der regelmäßig stattfindenden Monitoring-Besuche. Neben dem Quelldatenabgleich wird das Datenmonitoring den TZ als Ansprechpartner zur Verfügung stehen und in Rücksprache mit dem Datenmanagement TZ mit mangelnder Datenqualität identifizieren und in Zusammenarbeit mit dem betroffenen TZ für eine Verbesserung des Datenrückflusses sorgen.

\section{Elektronische Case Report Forms und Spender-Fragebögen}

Der Merkmalskatalog der Spender-Fragebögen und eCRFs wurden nach dem internationalen Metadatenstandard für klinische Studien: Operational Data Model (ODM) von der Organisation Clinical Data Interchange Standards Consortium (CDISC) modelliert. Die eCRFs und Spender-Fragbögen sind auf einer europäischen Forschungsinfrastruktur für medizinische Datenmodelle veröffentlicht (https://medical-data-models.org/34685) und in unterschiedlichen Dateiformaten zur Wiederverwendung verfügbar. Zur Unterstützung der semantischen Interoperabilität sind alle definierten Datenelemente semantisch annotiert durch Terminologie-Codes des Unified Medical Language Systems. Wann immer es möglich ist, wurden vorhandene Metadatendefinitionen internationaler Standards wiederverwendet und vorhandene Maßeinheiten mit dem Kodierungssystem Unified Code for Units of Measure (UCUM) kodiert.

Neben den Spender-Fragebögen zur Erfassung der physischen und psychischen Gesundheit werden medizinische Daten, die Rückschlüsse auf die Nierenfunktion geben, mittels eCRFs erhoben. Als klinische Variablen werden Daten verwendet, die im TZ bei den Vorund Nachsorgetermine zur LS routinemäßig und standardisiert erhoben werden, z. B. Serum-Kreatinin, systolischer und diastolischer Blutdruck. Die im Register verwendeten eCRFs enthalten neben den medizinischen Daten, die jedes Zentrum im Rahmen der Qualitätssicherung gesetzlich verpflichtend an das Institut für Qualitätssicherung und Transparenz im Gesundheitswesen (IQTIG) und Eurotransplant liefern muss, zusätzliche medizinische Daten zum Verlauf der Transplantation, der OP-Technik und weitere für die Transplantationsnephrologie wichtige Laborparameter. Die Spen- derfragebögen enthalten neben Fragen zur Einstellung zur LS und dem Verhältnis zum Empfänger auch Fragen zum sozioökonomischen und psychosozialen Hintergrund des LNS.

Die psychosozialen Daten des LNSs werden mittels standardisierter und etablierter Spender-Fragebögen in deutscher Sprache erhoben. Dies sind die Somatisierungs- (PHQ-15), Depressions(PHQ-9), Stress- (PHQ-S) und Ängstlichkeitsdimensionen (GAD-7) des Patients Healthy Questionnaires [16-18], das Multidimensionale Fatigue Inventar (MFI-20) [19], der SF-12 als Lebensqualitätsfragebogen [20], die Ambivalenzskala von Simmons [21] und die Resilienzskala RS-13 [22, 23]. Zusätzlich sind nicht-standardisierte Fragen zur Einstellung und Erwartung des Spenders bzgl. der Spende, seine Beziehung zum Empfänger und weitere spendenspezifische Fragen enthalten.

\section{Datenspeicherung}

Die pseudonymisierten MDAT werden nach dem Stand der Technik verschlüsselt übertragen und in der zentralen SOLKID-GNR Forschungsdatenbank gespeichert. Die IDAT werden getrennt beim Datentreuhänder am Zentrum für Informationsverarbeitung der WWU Münster gespeichert. Die Anforderungen der generischen Datenschutzkonzepte der Technologie- und Methodenplattform für die vernetzte medizinische Forschung e.V. (TMF) hinsichtlich des „Klinischen Moduls“ werden strikt befolgt und die Vorgaben der EU-DSGVO werden eingehalten [24].

\section{Datenprüfung und -bereinigung}

Datenqualitätsregeln zur schnellen Identifizierung nicht plausibler Daten wurden in der SOLKID-GNR Forschungsdatenbank implementiert und werden täglich über das Datenmanagement geprüft. Um eine ausreichende Datenqualität sicherzustellen werden zu regelmäßigen Zeitpunkten Datenexporte ausgewertet, wodurch eine umfangreiche Überprüfung von Plausibilitätsabweichungen, Außreißern und Datenvollständigkeit erfolgt.

\section{Datenauswertung}

Während der gesamten Laufzeit des Registers werden in regelmäßigen Abständen Berichte zum Rekrutierungsprozess, der Vollständigkeit der Daten, der Aktualität des Follow-up und der Qualität der Daten (z. B. Rate fehlender Werte, unplausible Dateneingaben) verfasst und veröffentlicht. 
Im dritten und fünften Jahr der 5-jährigen Realisierungsphase werden umfangreiche statistische Auswertungen zur Beantwortung konkreter wissenschaftlicher Fragestellungen durchgeführt. Dies beinhaltet insbesondere die präspezifizierten Hauptfragestellungen nach dem Einfluss der LS auf die psychosoziale und physische Gesundheit des Spenders sowie die Entwicklung eines spezifischen Risiko-Scores zur frühzeitigen Identifikation derjenigen LNS mit einem erhöhten bzw. zu hohen Risiko für ein negatives psychosoziales und/oder medizinisches Outcome. Weitere umfangreiche statistische Auswertungen werden nach Verstetigung des Registers durchgeführt, so dass die erhobenen Daten regelmäßig der Öffentlichkeit zugänglich gemacht werden.

\section{Datenarchivierung bzw. Vernichtung nach Projektabschluss}

Das Ziel von SOLKID-GNR ist die Einrichtung eines fortlaufenden Registers. Daher werden Daten erhoben und gespeichert ohne einen vordefinierten Endzeitpunkt der Datenlöschung. Falls möglich, sollen mit Hilfe des Registers Nachuntersuchungen bis zu 10-20 Jahre und länger nach LS möglich sein.

Sollte das Register entgegen der Planung nicht mehr fortgeführt werden können, werden die vorhandenen Daten vollständig anonymisiert, um weitere Datenauswertungen ohne Bezug zu den Personenstammdaten der Spender zu ermöglichen. Dieser Datensatz würde dann entsprechend der gesetzlichen Vorgaben 10 Jahre nach Projektende aufbewahrt.

\section{Datenfreigabe und Veröffentlichung}

Das SOLKID-GNR Konsortium orientiert sich an den FAIR-Prinzipien (Findable, Accessible, Interoperable and Reusable), um ein wissenschaftliches Datenmanagement und Datenfreigabe zu ermöglichen.

Den teilnehmenden TZ, sowie den beteiligten Patientenorganisationen wird nach Antrag und Begutachtung durch das Publikationskommittee ( $\triangleright$ Abb. 1) ermöglicht Datenauszüge für wissenschaftliche Projekte zu nutzen und zu veröffentlichen.

Personen oder Institutionen (wissenschaftlich oder öffentlich) die eine spezifische Fragestellung anhand der Registerdaten erarbeiten möchten, können einen Projekt-/Publikationsvorschlag beim Publikationskommittee einreichen. Das Publikationskommittee prüft den Antrag auf dessen wissenschaftliche Güte und ob Gründe gegen eine Freigabe sprechen, z. B. Publikationsbias oder Doppelpublikation. Zudem wird der Antrag auf inhaltliche Machbarkeit und Originalität geprüft. Nach positiver Prüfung können die zugehörigen Daten in anonymisierter Form nach einem zuvor abzuschließenden spezifischen Analysen- und Publikationsvertrages freigegeben werden.

\section{Datenschutz und rechtliche Aspekte}

Die zuständigen lokalen Datenschutzbeauftragten (DSB) der teilnehmenden TZ und der DSB der WWU Münster überwachen die Einhaltung relevanter Datenschutzauflagen, insbesondere der EUDSGVO.
Die Verarbeitungstätigkeit wurde erfasst, sowie eine Datenschutzfolgeabschätzung erstellt und werden fortlaufend aktualisiert.

\section{Ethik}

Die zustimmende Bewertung der zuständigen Ethikkommission der Ärztekammer Westfalen-Lippe und der Medizinischen Fakultät der Westfälischen Wilhelms-Universität Münster wurde am 24.01.2020 erteilt (2019-732-f-S). Anschließend wurden die jeweiligen zustimmenden Bewertungen der beteiligten Ethikkommissionen eingeholt, bevor die TZ mit dem Einschluss der LNS begonnen haben.

\section{Finanzierung des Registers und Nachhaltigkeit}

Während der 5-jährigen Realisierungsphase (01.03.201929.02.2024) wird das Register durch das BMBF (Förderkennzeichen: 01 GY1906) und der Medizinischen Fakultät der WWU Münster finanziert. Nach Abschluss der 5-jährigen Realisierungsphase wird die Medizinische Fakultät der WWU Münster das Register weiter finanzieren, womit eine Verstetigung des Registers gewährleistet ist.

\section{Stärken und Schwächen}

Eine Datenerhebung medizinischer Daten der LNS ist durch die Qualitätssicherung des IQTIG für drei Jahre nach erfolgter LS verpflichtend vorgesehen. Diese medizinischen Daten werden zukünftig an das Transplantationsregister übermittelt, so dass hier alle Datensätze zu LNS zusammengeführt und verwaltet werden. Psychosoziale Daten werden allerdings weder vom IQTIG noch vom Transplantationsregister berücksichtigt. Das SOLKID-GNR schließt diese Lücken in der Versorgungsforschung durch eine Langzeiterhebung medizinischer und psychosozialer Daten von LNS im deutschen Gesundheitssystem. Eine Vernetzung des SOLKID-GNR mit dem Transplantationsregister ist wünschenswert, um die angeführten Lücken zu schließen.

Eine wesentliche Stärke des Registers ist seine Interdisziplinarität und die enge Kooperation der drei TZ Münster, Hamburg jetzt Mainz und Heidelberg in der Konzeption und Realisierung. Gemeinsam mit allen teilnehmenden TZ wird in der Durchführung eine vollständige deutschlandweite Abdeckung erreicht.

Anhand der detaillierten prospektiven Analyse der Daten soll ein spezifischer Risiko-Score entwickelt werden, mittels dem bereits vor der Spende diejenigen LNS identifiziert werden, die ein erhöhtes bzw. zu hohes Risiko für einen negativen psychosozialen und medizinischen Outcome aufweisen. So kann das SOLKID-GNR zur stetigen Verbesserung der Versorgung der LNS in Deutschland beitragen.

Nach erfolgreicher Etablierung des SOLKID-GNR für die LS kann das Register in verschiedenen Aspekten (Aufbau, Struktur, Organisation der Abläufe, Datenerhebung, Verarbeitung, Management und Analyse) als „Blaupause“ für Lebendspende-Register anderer Organtransplantationen (Leber, Lunge, Ileum) dienen, die wegen des bestehenden Organmangels auch hier in Zukunft zunehmen 
werden. Die meisten dieser Lebendspenden werden an den gleichen TZ durchgeführt und werden einen anderen psychosozialen Einfluss auf die Lebendspender haben.

Eine Schwäche des SOLKID-GNR ist, dass es aktuell nur deutschsprachigen LNS möglich ist teilzunehmen. Um auch nicht-deutschsprachigen LNS die Teilnahme am Register zu ermöglichen werden zukünftig die Spender-Fragebögen, die die selbst-generierten Spender-Fragen enthalten, ebenfalls in anderen Sprachen zur Verfügung gestellt, so dass der Anteil an rekrutierbaren LNS erhöht wird.

Durch ein regelmäßig durchgeführtes internes Qualitätsmanagement werden Schwächen des Registers identifiziert und die Registerstruktur entsprechend angepasst, so dass das SOLKIDGNR konstant optimiert wird und die Belange der TZ berücksichtigt werden. So wird eine erfolgreiche Implementierung des SOLKID-GNR auch über die Realisierungsphase hinaus gewährleistet.

\section{Danksagung}

Die Autorinnen/Autoren bedanken sich bei den Mitarbeitern der SOLKID-GNR Registerzentrale und den teilnehmenden TZ für die Teilnahme am SOLKID-GNR. Zudem wird dem BMBF DLR-Projektträger für die Förderung des Registers gedankt (01GY1906).

\section{Interessenkonflikt}

Die Autorinnen/Autoren geben an, dass kein Interessenskonflikt besteht.

\section{Literatur}

[1] Sommerer C, Feuerstein D, Dikow R et al. Psychosocial and physical outcome following kidney donation-a retrospective analysis. Transpl Int 2015; 28: 416-428

[2] Kroencke S, Fischer L, Nashan B et al. A prospective study on living related kidney donors' quality of life in the first year: choosing appropriate reference data. Clin Transpl 2012; 26: E418-E427

[3] Meyer KB, Hartmann A, Mjoen G et al. Relationships Between Clinical, Self-Reported, and Donation Specific Outcomes: A Prospective Follow-up Study 10 Years After Kidney Donation. Ann Transplant 2017; 22: $148-155$

[4] Janki S, Klop KW, Dooper IM et al. More than a decade after live donor nephrectomy: a prospective cohort study. Transpl Int 2015; 28 : 1268-1275

[5] Gross CR, Messersmith EE, Hong BA et al. Health-related quality of life in kidney donors from the last five decades: results from the RELIVE study. Am J Transplant 2013; 13: 2924-2934

[6] Timmerman L, Laging M, Timman R et al. The impact of the donors' and recipients' medical complications on living kidney donors' mental health. Transpl Int 2016; 29: 589-602
[7] Clemens K, Boudville N, Dew MA et al. The long-term quality of life of living kidney donors: a multicenter cohort study. Am J Transplant 2011; 11: 463-469

[8] Wirken L, van Middendorp H, Hooghof CW et al. The Course and Predictors of Health-Related Quality of Life in Living Kidney Donors: A Systematic Review and Meta-Analysis. Am J Transplant 2015; 15: 3041-3054

[9] Lentine KL, Schnitzler MA, Xiao H et al. Depression diagnoses after living kidney donation: linking U.S. Registry data and administrative claims. Transplantation 2012; 94: 77-83

[10] Muzaale AD, Massie AB, Segev DL. Concerns about the long-term safety of live kidney donors are justified. Eur J Epidemiol 2017; 32: 91-93

[11] Mjoen G, Holdaas $\mathrm{H}$. Long term risk of mortality after living kidney donation. BMJ 2017; 357: j1770

[12] Mjoen G, Hallan S, Hartmann A et al. Long-term risks for kidney donors. Kidney Int 2014; 86: 162-167

[13] Suwelack B, Wormann V, Berger K et al. Investigation of the physica and psychosocial outcomes after living kidney donation - A multicentre cohort study (SoLKiD - Safety of Living Kidney Donors). BMC Nephrol 2018; 19: 83

[14] Harris PA, Taylor R, Minor BL Et al. The REDCap consortium: Building an international community of software platform partners. J Biomed Inform 2019; 95: 103208

[15] Lablans M, Borg A, Ückert F. A RESTful interface to pseudonymization services in modern web applications. BMC medical informatics and decision making 2015; 15: 2

[16] Kroenke K, Spitzer RL, Williams JB. The PHQ-9: validity of a brief depression severity measure. J Gen Intern Med 2001; 16: 606-613

[17] Kroenke K, Spitzer RL, Williams JB et al. The Patient Health Questionnaire Somatic, Anxiety, and Depressive Symptom Scales: a systematic review. Gen Hosp Psychiatry 2010; 32: 345-359

[18] Löwe B, Kroenke K, Herzog W et al. Measuring depression outcome with a brief self-report instrument: Sensitivity to change of the Patient Health Questionnaire (PHQ-9). Journal of Affective Disorder 2004; 81: 61-66

[19] Smets EM, Garssen B, Bonke B et al. The Multidimensional Fatigue Inventory (MFI) psychometric qualities of an instrument to assess fatigue. J Psychosom Res 1995; 39: 315-325

[20] Bullinger M, Kirchberger I, Ware ]. The German SF-36 Health Survey Translation and Psychometric Testing of a Generic Instrument for the Assessment of Health-Related Quality of Life. Z.f.Gesundheitswiss 1995; 3: 21-36

[21] Simmons RG, Marine SK, Simmons RL. Gift of life: the effect of organ transplantation on individual, family, and societal dynamics. New Brunswick, NJ: Transaction Publishers; 1987

[22] Leppert K, Koch B, Brähler E et al. Die Resilienzskala (RS) - Überprüfung der Langform RS-25 und einer Kurzform RS-13. Klin Diagnostik und Evaluation 2008; 1: 226-243

[23] Wagnild GM, Young HM. Development and psychometric evaluation of the Resilience Scale. J Nurs Meas 1993; 1: 165-178

[24] Pommerening K, Drepper J, Helbing K et al. Leitfaden zum Datenschutz in medizinischen Forschungsprojekten. Generische Lösungen der TMF 2.0. Berlin: Medizinisch Wissenschaftliche Verlagsgesellschaft; 2014 